\title{
ANÁLISE DA IMPRESSÃO 3D NO ENSINO DE ENGENHARIA: UM ESTUDO DE CASO NO LABORATÓRIO ABERTO DE BRASÍLIA
}

Jéssica Mendes Jorge (jessicamendes.jmj@gmail.com) - Universidade de Brasília/ Faculdade de Tecnologia/ Laboratório Aberto de Brasília

Alice Thadeu Firmino (alicethadeu@hotmail.com) - Universidade de Brasília/ Faculdade de Tecnologia/ Laboratório Aberto de Brasília

Andrea Cristina dos Santos (andreakieck@gmail.com) - Universidade de Brasília/ Faculdade de Tecnologia/ Laboratório Aberto de Brasília

\section{RESUMO}

A formação de profissionais que possam ser agentes de mudança na sociedade é enfatizada na literatura como fundamental para o desenvolvimento de um país. Desta forma, há um movimento das instituições de ensino para sair da abordagem tradicional e estimular um processo de aprendizado baseado na construção de conhecimento por meio da aplicação prática. Laboratórios de fabricação digital tem surgido nas instituições de ensino superior, em especial àquelas ligadas ao ensino de engenharia, utilizando as tecnologias de impressão 3D, permitindo a prototipagem de soluções para os problemas apresentados. Contudo, apesar da expansão da aplicação da impressão 3D no ensino, a mesma nem sempre vem acompanhada de uma metodologia de ensino. Neste contexto, este artigo discorre sobre um estudo de caso de como e onde a impressão 3D vem sendo aplicada no ensino de engenharia da Universidade de Brasília.

Palavras-chave: impressão 3D; ensino de engenharia; manufatura aditiva; laboratório aberto. 


\section{INTRODUÇÃ̃}

O enfoque para formação de um engenheiro com perfil empreendedor é colocado na literatura como fundamental para o desenvolvimento econômico e social de um país (ABDULWAHED, 2017; TAKS et al, 2017). Nesse sentido, os engenheiros podem ser agentes de mudança da sociedade e, por isso, devem pensar no contexto maior, na forma que buscam atuar no mercado de trabalho (GRECU et al, 2017).

Nessa perspectiva, existe uma mudança nas escolas de engenharia no mundo que seguem a estrutura de disciplinas tradicionais com currículos focados, principalmente, em ciências e teorias da engenharia, para um foco na direção da engenharia aplicada (TAKS et al, 2014; ABDULWAHED, 2017; GRECU, DENIS, 2017).

O conceito de Laboratórios Abertos (LA) se tornou conhecido a partir do surgimento dos laboratórios de fabricação digital - Fab Labs, que podem ser acadêmicos, profissionais ou públicos, filiados a uma rede mundial originada no Massachusetts Institute of Technology MIT (MIKHAK et al., 2002, FAB LAB, 20?).

Trazendo o conceito para uma abordagem mais centrada na educação superior, Gershenfeld (2008) define os FabLabs como ambientes pedagógicos que permitem indivíduos comuns resolverem seus próprios problemas através da fabricação. Essa iniciativa é uma das grandes responsáveis pela crescente tendência de implementação de espaços maker em instituições de ensino superior, em especial àquelas ligadas ao ensino de engenharia.

Uma das principais tecnologias utilizadas neste ambiente é a Manufatura Aditiva, mais especificamente as Impressoras 3D - I3D (tecnologia FDM -Fused Deposition Modeling, utilizando materiais poliméricos).

O baixo custo de aquisição e manutenção das I3D tem oferecido uma oportunidade para aplicação da manufatura aditiva além da prototipagem rápida (GONZALES, 2016). Ford e Minshall (2018) argumentam que a tecnologia I3D tem oferecido oportunidades para implementação de novas práticas de ensino, proporcionando a integração da resolução de problemas reais com o conteúdo apresentado em sala de aula. Os autores argumentam que apesar da expansão das I3D no ensino, as práticas de ensino permanecem incipientes carecendo de análises mais detalhadas.

Considerando este cenário, este trabalho discorre sobre como e onde a I3D integrada as metodologias ativas de ensino tem sido empregada no Laboratório Aberto de Brasília (LAB) da Faculdade de Tecnologia - Universidade de Brasília após um ano de funcionamento. 


\section{O EMPREGO DA IMPRESSÃO 3D NO ENSINO E ABORDAGEM PBL}

Uma pesquisa realizada por Simpson et al. (2017) apresenta as demandas da indústria para incorporação da Manufatura Aditiva. Por meio de um Workshop realizado nos EUA, envolvendo 66 representantes da academia, da indústria e do governo, foram levantados os principais tópicos referentes aos conhecimentos e habilidades relacionados a tecnologia de Manufatura Aditiva, sintetizadas na Tabela 1:

TABELA 1 - Tópicos de conhecimentos e habilidades relacionados a manufatura aditiva.

\begin{tabular}{|c|c|c|}
\hline Tópicos & Demandas & Assuntos relacionados \\
\hline $\begin{array}{l}\text { Manufatura } \\
\text { Aditiva }\end{array}$ & $\begin{array}{l}\text { Os estudantes terem uma visão de todos os } \\
\text { processos de MA e materiais relacionados, } \\
\text { capacidade de selecionar o processo de MA } \\
\text { pela variedade de aplicações. }\end{array}$ & $\begin{array}{l}\text { Termos, processos e tecnologias. Considerar o } \\
\text { modelo de negócio: cadeia de suprimentos, } \\
\text { economia, análise do ciclo de vida e } \\
\text { sustentabilidade. } \\
\text { Projeto para Manufatura aditiva. Tipos de } \\
\text { materiais e processos de fabricação. } \\
\text { Conhecimento sobre aplicações, sucesso e } \\
\text { carreiras na área. }\end{array}$ \\
\hline $\begin{array}{l}\text { Fundamentos } \\
\text { de } \\
\text { Engenharia }\end{array}$ & $\begin{array}{l}\text { Análises dos fundamentos da engenharia } \\
\text { com ênfase na ciência e seleção de } \\
\text { materiais (ser capaz de melhor } \\
\text { compreensão interações entre processo e } \\
\text { material) nas tecnologias de manufatura } \\
\text { (capaz de selecionar entre o processo de } \\
\text { fabricação tradicional e a manufatura } \\
\text { aditiva). }\end{array}$ & $\begin{array}{l}\text { Ciências materiais, mecanismo computacional, } \\
\text { metalurgia, seleção material, polímero, química. } \\
\text { Tecnologias de manufatura: visão dos processos } \\
\text { tradicionais (máquinas). } \\
\text { Metrologia, controle de qualidade, testes e } \\
\text { inspeção, verificação e validação. } \\
\text { Modelagem de engenharia, análise e estatística. } \\
\text { Pensamento sistêmico, mecatrônica, engenharia } \\
\text { econômica, programação, biomédica e } \\
\text { segurança. }\end{array}$ \\
\hline $\begin{array}{l}\text { Habilidades } \\
\text { profissionais }\end{array}$ & $\begin{array}{l}\text { A necessidade de preparar os estudantes } \\
\text { para resolver problemas e habilidades de } \\
\text { pensamento crítico melhor retirarem as } \\
\text { vantagens da tecnologia de manufatura } \\
\text { aditiva. Os participantes sugeriram incluir } \\
\text { no currículo empreendedorismo e o a } \\
\text { transferência tecnológica. }\end{array}$ & $\begin{array}{l}\text { Resolução de problemas e pensamento crítico. } \\
\text { Formação de equipes e colaboração, } \\
\text { Consciência cultural } \\
\text { Empreendedorismo, transferência de tecnologia; } \\
\text { Atendimento ao cliente e marketing } \\
\text { Aprender a "falhar e seguir em frente" } \\
\text { Alcançar amplitude e profundidade } \\
\text { Engenheiro ou um inventor } \\
\text { Tomada de decisão } \\
\text { Habilidades de ensino } \\
\text { Comunicação } \\
\text { Adaptabilidade }\end{array}$ \\
\hline $\begin{array}{l}\text { Projeto } \\
\text { (Design) }\end{array}$ & $\begin{array}{l}\text { Reconhecendo que a manufatura aditiva } \\
\text { reduz as restrições de projeto que são } \\
\text { tipicamente impostas pela manufatura } \\
\text { convencional, vários participantes } \\
\text { sugeriram a integração do projeto no } \\
\text { currículo. Sugerindo a introdução de } \\
\text { softwares de CAD, FEA, Organização } \\
\text { topológica e o processo de projeto. }\end{array}$ & $\begin{array}{l}\text { Software de CAX } \\
\text { Processo de projeto } \\
\text { Otimização topológica } \\
\text { Análise de elementos finitos } \\
\text { Desdobramento da Função Qualidade } \\
\text { Tolerância e dimensionamento geométrico } \\
\text { Engenharia reversa }\end{array}$ \\
\hline $\begin{array}{l}\text { Habilidades } \\
\text { criativas }\end{array}$ & $\begin{array}{l}\text { Relacionado a educação projeto muitos } \\
\text { participantes sugerem que é a oportunidade } \\
\text { para incluir no currículo técnicas de } \\
\text { criatividade e de design industrial. }\end{array}$ & $\begin{array}{l}\text { Artes, Design Industrial, Ideação, Técnicas de } \\
\text { Criatividade, Design Thinking }\end{array}$ \\
\hline
\end{tabular}

Fonte: Wimpson et al. (2017) 
Ford e Minshall (2018) colocam que a I3D é tipicamente usada em cinco diferentes contextos educacionais, escolas, universidades, espaços makers e na educação especial.

O ambiente de escolas envolve a educação básica, educação infantil, ensino fundamental e ensino médio. A I3D nesses níveis é utilizada para melhorar o entendimento do aluno em relação a conteúdos de matemática, física e química (FORD, MINSHALL, 2018). Como por exemplo, materialização do conhecimento, por meio da impressão de corpos dimensionais para entender geometria (CORUM, GAROFALO, 2015; HULEIHIL, 2017), até a prototipação de problemas reais, como por exemplo, a confecção de próteses de mão (COOK et al., 2015).

No contexto das universidades as atividades de I3D geralmente estão integradas aos conteúdos: computação gráfica, projeto e manufatura com polímeros, projeto de engenharia, projeto gráfico, engenharia industrial e negócio, informática, projeto mecânico, processo de manufatura, projeto de produto e desenvolvimento de produto (FORD, MINSHALL, 2018). Além de destas, envolve o próprio desenvolvimento das impressoras, com o desenvolvimento de modelos e controladores para $\mathrm{I} 3 \mathrm{D}$, integradas a metodologias ativas de ensino, mais especificamente a abordagem PBL (Project/Problem Base Learning - aprendizagem baseada em problemas/projetos)

A abordagem PBL estimula o aprendizado baseado na participação dos alunos em equipes multidisciplinares para a resolução de problemas reais, integrando a teoria com a prática. Fundamentada em princípios educacionais da pesquisa cognitiva, esta abordagem se difere da tradicional, caracterizada por uma recepção passiva e acumulação de conhecimentos por compreender a aprendizagem como um processo de construção do conhecimento (RIBEIRO, 2008).

Ribeiro (2005) argumenta que nos cursos de engenharia, tem um processo de resolução complexo que, em sua maioria, demanda a confecção de um artefato concreto, protótipo ou modelo. Desta forma, a abordagem PBL demanda uma maior dedicação dos alunos para desenvolverem as atividades de forma autônoma, necessitando de uma base de conhecimentos conceituais e técnicos, no período de desenvolvimento do projeto. 


\section{ANÁLISE DO LABORATÓRIO ABERTO DE BRASÍLIA - LAB}

O Laboratório Aberto de Brasília (LAB) é um programa da Faculdade de Tecnologia Universidade de Brasília, caracterizado por um ambiente colaborativo de aprendizagem ativa e multidisciplinar para o desenvolvimento e prototipação de produtos (ZIMMERMANN, 2018).

Desenvolvido durante o ano de 2017 e tendo sua operação iniciada em 2018, o LAB atua como: i) um agente de promoção das metodologias ativas no ensino, incentivando uma integração entre a teoria e a prática em disciplinas da engenharia e projetos de disciplinas de alunos da universidade, ii) um formador de profissionais com habilidades de I3D e iii) um prestador de serviços para a comunidade.

O LAB foi idealizado para atender os sete princípios comuns às metodologias ativas de ensino, apresentados por Diesel et al (2017): i) o processo de aprendizagem é centrado no aluno, ii) o estudante é protagonista do seu aprendizado, iii) o pensamento crítico é estimulado na sala de aula por meio de casos reais, iv) o trabalho em equipe é visto como elemento de mobilização para a inovação e construção de conhecimento, v) o professor tem o papel de facilitador desse processo.

Para analisar a integração das metodologias ativas com a impressão 3D no LAB, inicialmente baseou-se no trabalho de Simpson et al. (2017) no qual buscou-se identificar como e onde a I3D utilizada. Para isso fez-se o levantamento de todas as atividades executadas no LAB no último ano.

Com base nestas informações, elaborou-se em uma pirâmide com quatro níveis diferentes, considerando o aprofundamento do conteúdo de I3D (conhecimento e habilidades) e a abordagem de ensino utilizada, ilustrada na Figura 1.

Na base da pirâmide estão os estudantes de ensino médio e fundamental, professores e profissionais, no segundo nível os estudantes dos cursos de engenharia, as equipes de competição e os projetos de pesquisa estão representados no terceiro nível, a equipe interna do LAB está no quarto nível da pirâmide. 
Figura 1 - Onde e como a impressão 3D é aplicada no LAB.

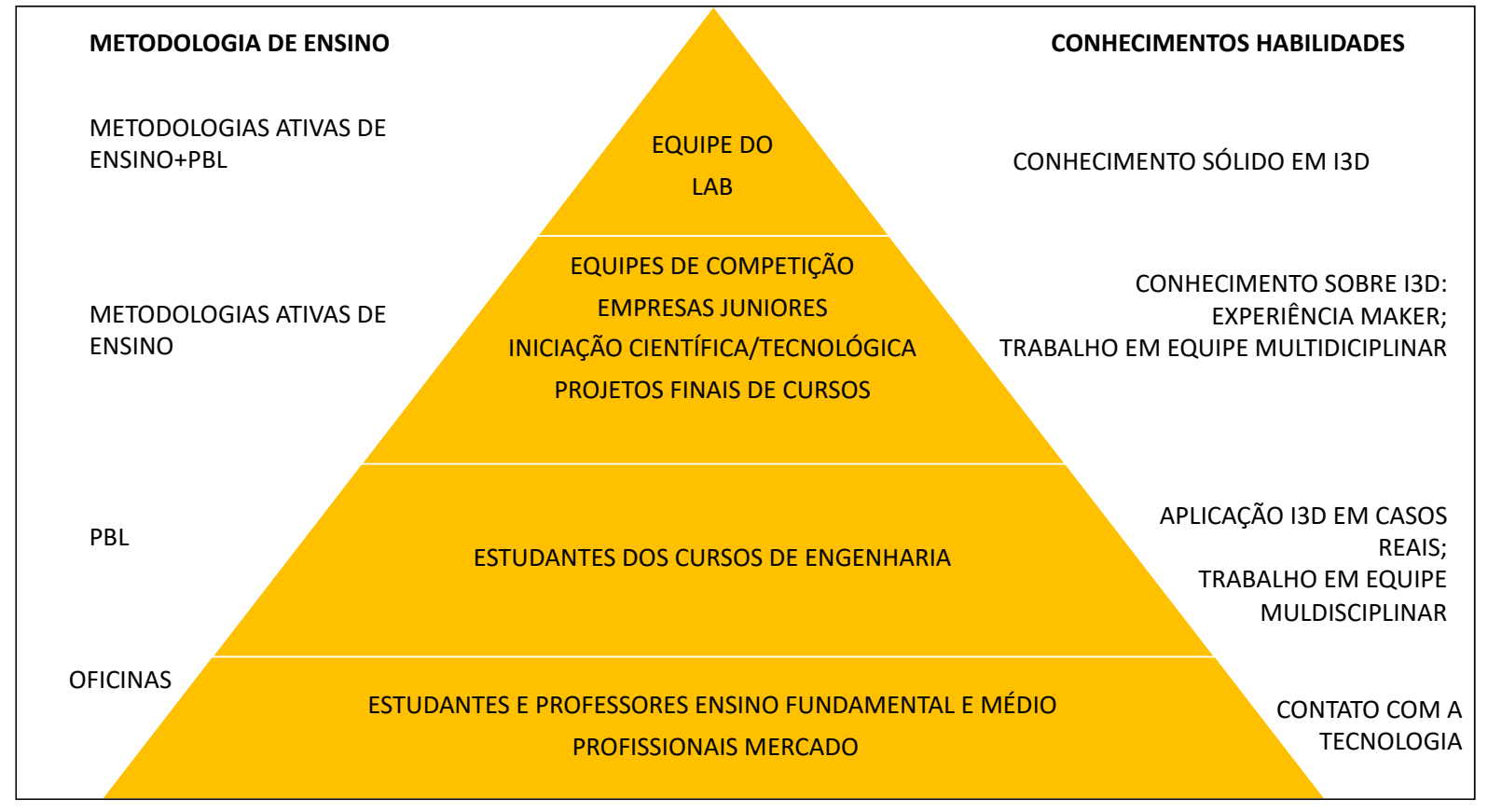

Na base da pirâmide estão os estudantes e professores do ensino fundamental e médio, e os professionais de mercado. A metodologia de ensino utilizada para este público são oficinas sobre a I3D a fim de proporcionar um contato com a tecnologia. A Tabela 3 sintetiza as atividades realizadas para esse público no primeiro ano de operação do LAB.

Tabela 3 - Atividades de I3D aplicadas no ensino fundamental, médio e profissionais de mercado.

\begin{tabular}{|c|c|c|c|}
\hline \multirow{2}{*}{$\begin{array}{c}\text { Conteúdo } \\
\text { Técnico } \\
\text { Ministrado }\end{array}$} & \multirow{2}{*}{$\begin{array}{l}\text { Conhecimento e } \\
\text { habilidades } \\
\text { desenvolvidas }\end{array}$} & \multicolumn{2}{|c|}{ Externo } \\
\hline & & Escolas & Profissional \\
\hline $\begin{array}{l}\text { Processo de } \\
\text { Manufatura }\end{array}$ & $\begin{array}{l}\text { Conhecimento sobre as } \\
\text { aplicações da I3D }\end{array}$ & $\begin{array}{l}\text { Meninas Velozes (Projeto } \\
\text { ensino Médio) }\end{array}$ & $\begin{array}{l}\text { Oficinas público externo }- \text { não } \\
\text { alunos }\end{array}$ \\
\hline Aditiva - I3D & $\begin{array}{l}\text { Conhecimento sobre os } \\
\text { processos da I3D }\end{array}$ & $\begin{array}{l}\text { Oficinas para professores do } \\
\text { INSTEI (Centro de Ensino } \\
\text { Ceilândia Norte). }\end{array}$ & \\
\hline
\end{tabular}

No segundo nível da pirâmide estão os estudantes dos cursos de engenharia da Universidade de Brasília (UnB). As metodologias de ensino aplicada é o PBL, a I3D proporcionar aos alunos aplicação do conhecimento adquirido em sala de aula em problemas 
reais, tendo contato com a I3D para materializar as soluções propostas. A Tabela 4 sintetiza as atividades de I3D aplicadas nesse nível.

Tabela 4 - Atividades de I3D aplicadas nos cursos de engenharia.

\begin{tabular}{|c|c|c|}
\hline $\begin{array}{l}\text { Conteúdo Técnico } \\
\text { Ministrado }\end{array}$ & $\begin{array}{c}\text { Conhecimento e } \\
\text { habilidades desenvolvidas }\end{array}$ & Interno UnB \\
\hline \multirow{2}{*}{$\begin{array}{l}\text { Processo de Manufatura } \\
\text { Aditiva-I3D }\end{array}$} & \multirow{4}{*}{$\begin{array}{llr}\text { Conhecimento } & \text { sobre } & \text { as } \\
\text { aplicações da } & \text { I3D } \\
\text { Conhecimento sobre } & \text { os } \\
\text { processos da I3D } & \\
\text { Resolução de problemas } & \\
\text { Pensamento crítico }\end{array}$} & Disciplina de Vibrações (Eng. Mecânica) \\
\hline & & $\begin{array}{l}\text { Disciplina de Engenharia de Produto (Eng. } \\
\text { Produção) }\end{array}$ \\
\hline \multirow{3}{*}{$\begin{array}{l}\text { Projeto para Manufatura } \\
\text { Aditiva (tipos de materiais e } \\
\text { processos de fabricação) }\end{array}$} & & $\begin{array}{l}\text { Disciplina Optativa Tecnologias Assistivas } \\
\text { (Optativa) }\end{array}$ \\
\hline & & Disciplina de projeto integrador (Optativa) \\
\hline & $\begin{array}{l}\text { Formação de equipes e } \\
\text { colaboração }\end{array}$ & $\begin{array}{l}\text { Disciplina de Desenho Mecânico Assistido } \\
\text { por Computador (Engenharias) }\end{array}$ \\
\hline Resolução de problemas & & $\begin{array}{l}\text { Disciplina de Desenho Industrial Assistido } \\
\text { por Computador (Engenharias) }\end{array}$ \\
\hline
\end{tabular}

No nível três (Figura 1) estão as equipes de competição, empresas juniores, iniciação científica/tecnológicas e os projetos de final de curso. Neste grupo, a experiência maker e a principal característica, para isso eles necessitam de conhecimentos sobre a impressão 3D, processo de manufatura e o projeto para manufatura aditiva. Destaca-se uma interação muito grande deste nível com o nível 4, tornando o trabalho mesmo de iniciação científica mais multidisciplinar. A Tabela 5 sintetiza as atividades de I3D aplicadas nesse nível.

Tabela 5 - Atividades de I3D desenvolvidas com as equipes de competição, empresas juniores, iniciação científica e projetos de final de curso.

\begin{tabular}{|c|c|c|}
\hline $\begin{array}{l}\text { Conteúdo Técnico } \\
\text { Ministrado }\end{array}$ & $\begin{array}{l}\text { Habilidades } \\
\text { desenvolvidas }\end{array}$ & Interno UnB \\
\hline $\begin{array}{l}\text { Processo de Manufatura } \\
\text { Aditiva-I3D }\end{array}$ & $\begin{array}{l}\text { Conhecimento sobre as } \\
\text { aplicações da I3D }\end{array}$ & $\begin{array}{l}\text { Empresas Juniores com protótipos na área de } \\
\text { engenharia mecânica }\end{array}$ \\
\hline \multirow{4}{*}{$\begin{array}{l}\text { Projeto para Manufatura } \\
\text { Aditiva (tipos de materiais } \\
\text { e processos de fabricação) }\end{array}$} & \multirow{4}{*}{$\begin{array}{l}\text { Conhecimento sobre os } \\
\text { processos da I3D } \\
\text { Resolução de } \\
\text { problemas }\end{array}$} & $\begin{array}{l}\text { Equipes de competição nas áreas de automotiva, } \\
\text { aeroespacial e robótica. }\end{array}$ \\
\hline & & Protótipos na área da saúde \\
\hline & & Protótipos na área de energia \\
\hline & & tecnologias assistivas \\
\hline
\end{tabular}


No topo da pirâmide (Figura 1) é apresentada a equipe do LAB, que é multidisciplinar e composta por alunos diferentes cursos de engenharia (mecânica, produção, mecatrônica, elétrica, automotiva - atualmente a equipe conta com 20 integrantes). A metodologia de aprendizado é uma combinação das metodologias ativas de ensino e de PBL. As demandas são projetos internos da UnB (pesquisa, final de curso, equipes de competição ou outro) e externos a UnB (outras universidades, empresas, startups) fazem com que haja pelo menos dois alunos de diferentes cursos envolvidos.

Todas as demandas são tratadas como serviços do LAB. Sendo classificadas em três tipos:

- $\quad$ Projeto: a equipe $\mathrm{LAB}$ desenvolve uma solução para um problema real apresentado pelo cliente.

- $\quad$ Modelagem: a equipe desenvolve um modelo em 3D para uma solução que está em outra extensão, podendo fazer uma análise técnica da peça e entregando o arquivo com correções e melhorias para o cenário em que será utilizada.

- Impressão 3D: a equipe imprime o modelo em 3D apresentado pelo cliente, podendo este ser um protótipo ou um produto final.

Além da prestação de serviços, a equipe interna atua na montagem e manutenção das impressoras 3D, preparação e realização de workshops e oficinas. A Tabela 6 sintetiza as atividades de I3D desenvolvidas nesse nível.

Tabela 6 - Atividades de I3D desenvolvidas pela Equipe do LAB.

\begin{tabular}{|c|c|c|c|}
\hline Cont & Habilidades desenvolvidas & Interno UnB & Externo \\
\hline \multirow{6}{*}{$\begin{array}{l}\text { Processo de Manufatura } \\
\text { Aditiva - I3D. } \\
\text { Aplicações I3D } \\
\text { Projeto para Manufatura } \\
\text { Aditiva (tipos de } \\
\text { materiais e processos de } \\
\text { fabricação) } \\
\text { Modelagem em softwares } \\
\text { CAD e outros } \\
\text { Técnicas de criatividade } \\
\text { Manutenção de I3D }\end{array}$} & \multirow{6}{*}{$\begin{array}{l}\text { Resolução de problemas } \\
\text { Pensamento crítico } \\
\text { Pensamento criativo } \\
\text { Formação de equipes } \\
\text { multidisciplinares } \\
\text { Colaboração } \\
\text { Responsabilidade } \\
\text { Pro-atividade } \\
\text { Curiosidade dos } \\
\text { Cumprimento } \\
\text { procedimentos operacionais } \\
\text { Regras de convívio espaço } \\
\text { compartilhado } \\
\text { Regras de segurança } \\
\text { Flexibilidade - atividades } \\
\text { rotativas }\end{array}$} & $\begin{array}{l}\text { Suporte às disciplinas } \\
\text { para prototipagem em } \\
\text { I3D }\end{array}$ & $\begin{array}{l}\text { Serviços de } \\
\text { prototipagem em I3D } \\
\text { para terceiros }\end{array}$ \\
\hline & & Workshops de I3D & $\begin{array}{l}\text { Projetos, modelagem } \\
\text { e I3D }\end{array}$ \\
\hline & & Oficinas de I3D & Workshops de I3D \\
\hline & & $\begin{array}{l}\text { Montagem } \\
\text { impressora 3D }\end{array}$ & $\begin{array}{l}\text { Montagem } \\
\text { impressora 3D }\end{array}$ \\
\hline & & $\begin{array}{l}\text { Manutenção de das } \\
\text { impressoras 3D }\end{array}$ & Oficinas I3D \\
\hline & & $\begin{array}{l}\text { Realização de desafios } \\
\text { de prototipagem }\end{array}$ & \\
\hline
\end{tabular}


Em seu primeiro ano de implementação, o LAB contribuiu com a formação de quatro alunos (uma engenheira de produção, dois engenheiros mecânicos e um engenheiro mecatrônico) que estão inseridos no mercado de trabalho atuando com manufatura aditiva. Para o final do seu segundo ano de operação, o LAB tem uma previsão de contribuir com a formação de 15 alunos, que atualmente compõem a equipe do LAB.

\section{CONCLUSÃO}

O artigo apresentado surge do contexto no qual o desenvolvimento econômico e social demanda profissionais de engenharia com perfil empreendedor que, além de conhecer e dominar as novas tecnologias, também sejam dotados de habilidades sociais, pessoais, metodológicas e técnicas.

Desta forma, há um movimento das instituições de ensino para integrar as tecnologias habilitadoras com metodologias de ensino no processo de formação dos alunos, tanto no ensino básico como nas universidades. Por ser de baixo custo, a I3D tem sido uma das tecnologias habilitadoras mais adotadas nessa revolução do ensino, expandindo sua aplicação para além da da prototipagem rápida comumente empregadas.

Neste trabalho foi apresentado o estudo de caso sobre o Laboratório Aberto de Brasília - LAB, um programa da Universidade de Brasília que incentiva as metodologias ativas de ensino, utilizando a tecnologia de Impressão 3D, em quatro níveis de aplicação: estudantes do ensino fundamental e médio, aplicando oficinas de aprendizagem; estudantes dos cursos de engenharia; as equipes de competição, empresas juniores, projetos de iniciação científica/tecnológica e projetos de final de curso, utilizando as metodologias ativas de ensino; os alunos que formam a equipe do LAB, combinando os modelos do PBL com as metodologias ativas de ensino.

A estratégia de uso da tecnologia de impressão 3D no LAB tem oferecido a oportunidade de desenvolver habilidades técnicas, metodológicas, sociais e pessoais. Contudo, observa-se que o processo de acompanhamento do desenvolvimento dos conhecimentos habilidades desenvolvidos no LAB precisa ser aprimorado. Na tabela 7 estão sintetizados as categorias, os conhecimentos e habilidades e seu respectivo contexto, a serem aprimorados e avaliados. 
Tabela 7 - Conhecimentos e habilidades desenvolvidas pelo LAB.

\begin{tabular}{|c|c|c|}
\hline Cat & $\begin{array}{c}\text { Conhecimentos e habilidades a serem } \\
\text { desenvolvidas }\end{array}$ & Contexto \\
\hline \multirow{5}{*}{ 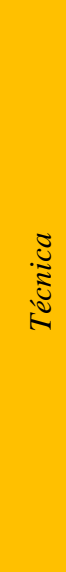 } & $\begin{array}{l}\text { Conhecimento dos fundamentos básicos e } \\
\text { de todos os processos de manufatura aditiva } \\
\text { (MA) }\end{array}$ & $\begin{array}{l}\text { Ser capaz de selecionar efetivamente o processo de MA para uma } \\
\text { variedade de aplicações. }\end{array}$ \\
\hline & Conhecimento em ciência dos materiais & Ser capaz de entender as interações do processo com o material. \\
\hline & $\begin{array}{l}\text { Conhecimento do processo de projeto e } \\
\text { desenvolvimento de produto }\end{array}$ & \multirow[b]{2}{*}{$\begin{array}{l}\text { Ser capaz de utilizar softwares de modelagem e ter conhecimento de } \\
\text { todo o processo de projeto e desenvolvimento de um produto. }\end{array}$} \\
\hline & $\begin{array}{l}\text { Conhecimento de ferramentas de design } \\
\text { digital (CAD, FEA, otimização de } \\
\text { softwares) }\end{array}$ & \\
\hline & O estado da arte do conhecimento & $\begin{array}{l}\text { Devido ao crescimento das responsabilidades de trabalho, o } \\
\text { conhecimento tem ganhado cada vez mais importância (nível mais alto } \\
\text { de desenvolvimento). }\end{array}$ \\
\hline \multirow{5}{*}{$\begin{array}{l}0 \\
0 \\
0 \\
10 \\
0 \\
0 \\
0 \\
0 \\
0 \\
2 \\
2\end{array}$} & Resolução de Problemas & Utilização da MA para resolver problemas de engenharia. \\
\hline & Pensamento crítico & Avaliar criticamente a operação e manutenção de sistemas. \\
\hline & Criatividade integrada ao design Industrial & Pensamento criativo com foco em soluções industriais inovadoras. \\
\hline & $\begin{array}{l}\text { Planejar, supervisionar, elaborar e } \\
\text { coordenar projetos e serviços de engenharia }\end{array}$ & Projetar e conduzir experimentos interpretando resultados. \\
\hline & $\begin{array}{l}\text { Avaliar o impacto das atividades da } \\
\text { engenharia no contexto social e ambiental }\end{array}$ & $\begin{array}{l}\text { Visão macro do impacto gerado pelo processe e de toda a cadeia } \\
\text { produtiva. }\end{array}$ \\
\hline \multirow{5}{*}{ 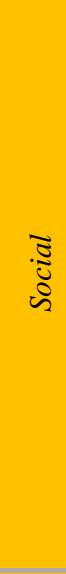 } & Trabalho em equipe & Atuar em equipes multidisciplinares. \\
\hline & $\begin{array}{l}\text { Comunicar-se efetivamente (escrita, oral, } \\
\text { gráficos) }\end{array}$ & $\begin{array}{l}\text { Habilidade de filtrar o desnecessário e repassar e escolher o canal } \\
\text { adequado a ser utilizado dentro de qualquer contexto. }\end{array}$ \\
\hline & $\begin{array}{l}\text { Compreender e aplicar a ética e a } \\
\text { responsabilidade profissional }\end{array}$ & Imperativos de conduta que formam a consciência do profissional. \\
\hline & Habilidade de transferir conhecimento & $\begin{array}{l}\text { De acordo com as atuais mudanças demográficas, conhecimentos } \\
\text { tácitos e explícitos precisam ser trocados, pois cada vez mais as } \\
\text { organizações precisam reter o conhecimento dentro e não somente nas } \\
\text { pessoas. }\end{array}$ \\
\hline & Habilidades de liderança & Atividades com maiores responsabilidades. \\
\hline \multirow{4}{*}{$\begin{array}{l}\widetilde{\Xi} \\
\vdots \\
\vdots \\
\vdots \\
2\end{array}$} & Flexibilidade & $\begin{array}{l}\text { Flexibilidade com as responsabilidades concedidas desvio a atividades } \\
\text { de trabalho rotativas. }\end{array}$ \\
\hline & Habilidade de trabalhar sobre pressão & $\begin{array}{l}\text { Menor ciclo de vida do produto e redução do tempo de comercialização } \\
\text { tem gerado maior necessidade de inovação e gerar resultados sob } \\
\text { pressão. }\end{array}$ \\
\hline & Mindset Sustentável & Mentalidade direcionada para iniciativas e soluções sustentáveis. \\
\hline & Complaplience & Cumprir-se e fazer cumprir as normas legais. \\
\hline
\end{tabular}




\section{REFERÊNCIAS}

ABDULWAHED, M. Technology Innovation and Engineering Education and Entrepreneurship (TIEE) in Engineering Schools: Novel Model for Elevating National Knowledge Based Economy and Socio-Economic Sustainable Development. Sustainability v. 9, n. 171, 2017.

COOK, K. L., BUSH, S.B., COX, R. Creating a prosthetic hand: 3D printers innovate and inspire and maker movement, Sci. Child 53 (2015) 80-86.

DIESEL, A.; BALDEZ, A. L. S.; MARTINS, S. N. Os princípios das metodologias ativas de ensino: uma abordagem teórica. Revista Thema, v. 14, n. 1, p. 268-288, 2017.

FAB LAB site, http://www.fabfoundation.org/index.php/fab-labs/index.html. Acesso em 15 de setembro de 2017. LABORATÓRIOS ABERTOS. SENAI. 2015. site: http://www.portaldaindustria.com.br/senai/canais/inovacaoe-tecnologia/laboratorios-abertos-senai/laboratorios-abertos-senai-quem-somos/

FORD, S., MINISHALL, T. Invited review article: Where and how 3D printing is used in teaching and education. Additive Manufacturing, v. 19 n. 1, p. 131-150, 2019.

GERSHENFELD, N. Fab: the coming revolution on your desktop -from personal computers to personal fabrication. Basic Books, 2008.

GONZALES, M. A. C. Ferramenta para concepção, projeto e operação de espaços para ensino de engenharia que incentivem a criatividade e a inovação. Dissertação. Escola Politécnica da Universidade de São Paulo. 2016. $225 \mathrm{p}$.

GRECU, V.; DENIS, C. Benefits of entrepreneurship education and training for engineering students. In: MATEC Web of Conference 121, 12, 2017.

HULEIHIL, M. 3D printing technology as innovative tool for math and geometry teaching applications, 5th Glob. Conf. Mater. Sci. Eng. (2017).

RIBEIRO, L. R. C. Aprendizagem baseada em problemas (PBL) na educação em Engenharia. Revista de Ensino de Engenharia, v. 27, p. 23-32, 2008.

RIBEIRO, L. R.C. A Aprendizagem Baseada em Problemas (PBL): uma implementação na educação em engenharia na voz dos atores. Tese de Doutorado da Universidade Federal de São Carlos, São Carlos, 2005.

TAKS, M.; TYNJALA, P.; TODING, M. Engineering Students' Experiences in Studying Entrepreneurship. Journal of Engineering Education, v. 103, n. 4, p. 573-598, 2014.

ZIMMERMANN, A. C. Proposição de ambiente de aprendizagem ativa: Laboratório Aberto de Brasília. 2018. 155 f., il. Trabalho de Conclusão de Curso (Bacharelado em Engenharia de Produção) - Universidade de Brasília, Brasília, 2018. 